\title{
Kurz angewendete Interventionen zur Angstbewältigung bei Kletteranfängern wirken nicht
}

\author{
Stefan Künzell ${ }^{1,2}$ und Vanessa Mann ${ }^{1}$ \\ 'Justus-Liebig-Universität Gießen \\ ${ }^{2}$ Universität der Bundeswehr, Neubiberg
}

\begin{abstract}
Zusammenfassung. Hilft eine kurz applizierte kognitive Technik oder ein schnell durchgeführtes Entspannungsverfahren Kletteranfängern bei der Angstbewältigung? Um dies zu untersuchen, wendeten wir bei Kletteranfängern vor dem Erklettern der ersten Route das Selbstinstruktionstraining nach Meichenbaum (1977a, 1977b) oder die Deep Breathing Technik nach Lichstein (1988) an. Die Zustandsangst wurde über ein Angst-Thermometer gemessen (Houtman \& Bakker, 1989), als zusätzliche Hinweise auf Angst dienten Herzfrequenz und Klettergeschwindigkeit (vgl. Pijpers, Oudejans, Holsheimer \& Bakker, 2003). Die Herzfrequenz sank unmittelbar nach der Instruktion, was auf die Wirksamkeit der Manipulation hinweist. Beide Techniken zeigten jedoch gegenüber der Kontrollgruppe während des Kletterns keine Wirkung. Zwar stiegen die Werte auf dem Angstthermometer mit zunehmender Höhe an, die beiden Treatmentgruppen unterschieden sich aber nicht von der Kontrollgruppe. Nur kurzfristig eingesetzte Verfahren zur Angstreduktion haben also keine Wirkung. Eine langfristige Vorbereitung sowie ein Training der Intervention scheinen für die Angstreduktion nötig zu sein.

Schlüsselwörter: Angst, Sportklettern, Selbstinstruktion, Entspannungsverfahren
\end{abstract}

Brief interventions to control anxiety do not work

Abstract. Do either instantly applied relaxation methods or a quickly executed cognitive method help climbing novices to control their state anxiety? We used Lichstein's (1988) Deep Breathing Technique or Meichenbaum's (1977a, 1977b) Self Instruction Training technique as methods to reduce anxiety in climbing novices immediately before climbing their first route. State anxiety was measured by using an anxiety thermometer (Houtman \& Bakker, 1989); as additional indicators of anxiety we measured heart rate and climbing velocity (see Pijpers et al., 2003). The heart rate decreased after the instructions were given, which shows the efficacy of the manipulation. Compared to a control group, neither of the techniques showed anxiety-reducing effects for the novices during climbing. Therefore, instantly applied manipulations for reducing anxiety do not seem to have an effect, and long-term preparation as well as training of the interventions seem to be necessary for anxiety reduction.

Key words: anxiety, sport climbing, self-instruction, deep-breathing technique

\section{Angst und Angstbewältigung im Sport}

Angst hat im Sport eine notwendige, Verletzungen minimierende und eine schädliche, leistungshemmende Wirkung (vgl. Schack, 1997). Sinnvoll und eventuell lebensrettend ist es, aus ,,angepasster" Angst das Verhalten abzuwägen und die Aufmerksamkeit auf bestimmte gefährliche Aspekte des eigenen Handelns zu lenken. Dies kann dazu führen, dass beispielsweise in einem lawinengefährdeten Gebiet eine Skitour abgebrochen wird oder aber der Aufstieg mit Entlastungsabständen durchgeführt wird. „Angepasst“ ist die Angst, wenn die subjektiv empfundene Bedrohung der objektiven Bedrohung entspricht. „Unangepasst" ist die Angst, wenn der subjektiv empfundenen Bedrohung keine oder nur eine geringe objektive Bedrohung zu Grunde liegt. Beim Klettern - vor allem im Anfängerbereich im Top-Rope-Klettern - überwiegt diese unangepasste Angst. Beim Top-RopeKlettern ist der Kletterer in ein Seil eingebunden, das 
über einen Umlenker, der sich am Ende der Route befindet, zu dem Sicherer geführt wird. Das Sicherungsseil kommt also immer von oben (daher top rope) und wird vom Sicherer straff gehalten. Im Falle eines Sturzes entspricht die Fallhöhe der Seildehnung, der Sturz ist weich, und bei richtiger Handhabung besteht kein Verletzungsrisiko. Die objektive Gefahr ist also nahezu Null, während die subjektiv erlebte Gefahr groß werden kann. In verschiedenen Studien konnte nachgewiesen werden, dass Angst beim Klettern sowohl die flüssige Ausführung der Bewegungen (Pijpers et al., 2003), die Griffstärke (Scherer, Kuhn, Henn \& Zepig, 2010) als auch die visuelle Aufmerksamkeit (Nieuwenhuys, Pijpers, Oudejans \& Bakker, 2008) beeinträchtigt. Insofern bietet es sich an, durch psychologische Maßnahmen zu versuchen, die Angst bei Kletteranfängern zu reduzieren.

Angst zeigt sich in drei Erscheinungsformen: Durch eine somatische oder (neuro-)physiologische Komponente (erhöhte Herzfrequenz, Schwitzen, Änderung des Atemrhythmus etc.), durch eine subjektive, kognitive Komponente (Besorgnis) sowie durch Mimik und Verhalten (z. B. Hackfort \& Schwenkmezger, 1980; Lazarus-Mainka \& Siebeneick, 2000). Angst kann direkt durch einen bedrohlichen Reiz ausgelöst werden (Zajonc, 1984), es kann aber auch erst eine kognitive Bewertung des Reizes notwendig sein (Lazarus, 1984). Es hängt wohl von den Umständen $\mathrm{ab}$, welche der beiden Prozesse ursächlich und welche Begleiterscheinung sind. Neurobiologisch betrachtet, korreliert Angst mit einer Aktivität der Amygdala. Auf einem schnellen, nicht bewussten Weg entsteht diese Aktivität aus dem sensorischen Angstreiz direkt über dem Thalamus, auf einem langsamen, bewussten Weg vom Thalamus über den sensorischen und den präfrontalen Kortex (LeDoux, 1997; Myers, 2008; van Reekum et al., 2007).

Wissenschaftlich untersuchte Verfahren der Angstregulation stammen vorrangig aus der klinischen Psychologie und können nicht immer im Sport eingesetzt werden. Dabei ist eine Vielzahl von Verfahren vorzufinden. Analog zur Angstentstehung können auch bei der Angstregulation kognitive und somatische Verfahren (Baumann, 2006; Lichstein, 1988) eingesetzt werden. In der vorliegenden Studie werden als somatisches Verfahren eine Atemübung nach Lichstein (1988) und als kognitives Verfahren das Selbstinstruktionstraining nach Meichenbaum (1977a, 1977b) eingesetzt.

\section{Atemübungen}

Neben der physiologischen Funktion für den Gesamtzustand ist die Atmung auch ein Indikator für die psychische Verfassung eines Menschen. Jegliche Gemüts- bzw. Zustandsveränderung spiegelt sich in der
Veränderung der Atmung, in Bezug auf Atemtiefe und Atemfrequenz, wider. Pressatmung, Hyperventilation, Atemlosigkeit vor Schreck, tiefes Atmen vor einer Gefahr sind Beispiele für Atemveränderungen, psychische Anspannung beeinflusst die Art und Weise, wie wir atmen. Dieser Prozess kann auch umgekehrt stattfinden: Durch tiefes Atmen kann eine Reduktion der psychischen und physischen Anspannung erfolgen, wodurch sich Körper und Geist wieder entspannen können (vgl. Olschewski, 1995).

In der vorliegenden Studie wurde mit einer der Versuchsgruppen eine Technik durchgeführt, die sich Deep Breathing Technik nennt und zu den Brief Relaxation Methods gehört (Lichstein, 1988). Wie der Name schon zeigt, handelt es sich hier um eine Atemtechnik, die schnell und dezent überall durchgeführt werden kann und keine ausführlichen Erklärungen benötigt. Diese Art von Atemtechnik erfreut sich besonders im sportlichen Kontext großer Beliebtheit, da in kürzester Zeit ein moderates Level der Entspannung erreicht werden kann und sie besonders bei emotionalen Belastungen wie Ärger und Angst geeignet ist. Die Deep Breathing Technik unterteilt sich dabei in drei Phasen: Tiefes Einatmen, Anhalten des Atems, langsames Ausatmen. Jede Phase dient der Gesamtwirksamkeit der Technik. Durch das Einatmen und das Konzentrieren auf den Atem soll von unliebsamen Gedanken abgelenkt werden; Luftanhalten erhöht das Kohlenstoffdioxidlevel, was zu einer leichten Lethargie führt und das anschließende Ausatmen bewirkt eine Reduktion der Anspannungen in der Muskulatur (vgl. Baumann, 2006; Lichstein, 1988).

Die Wirksamkeit von Atemübungen konnte von Bell und Saltikov (2000) und Sudsuang, Chentanez und Veluvan (1991) nachgewiesen werden. Wie Atemübungen jedoch genauer auf den Organismus wirken, ist umstritten. Während Lum (1983) von einer schlichtweg regulativen Funktion ausgeht, die die gestörte Atmung in Situationen emotionaler Angespanntheit wieder reguliert, gehen Garssen, De Ruiter und Van Dyke (1992) davon aus, dass Atemübungen zur Entspannung beitragen, weil sie die Aufmerksamkeit auf sich ziehen (vgl. Payne, 2004).

\section{Selbstinstruktionstraining nach Meichenbaum}

Nach Munzert (1997) kommt der Sprache, besonders in motorischen Lernprozessen, eine begleitende und unterstützende Funktion zu. Die Selbstinstruktion gilt als eine von mehreren Methoden der Verhaltensmodifikation. Ziel ist es hierbei, ein Modellverfahren zu erarbeiten und in sprachlichen Anweisungen zu formulieren, so dass die Personen sich in Angstsituationen solcher Anweisungen bedienen und sich innere 
Instruktionen zur Angstkontrolle geben können (vgl. Hackfort \& Schwenkmezger, 1980; Meichenbaum, 1977b). Charakterisiert wird das Verfahren der Selbstinstruktion durch vier Elemente: Der Problembestimmung (Was ist zu tun?), der Aufmerksamkeits- und Verhaltensanleitung (Was ist zu beachten? Wie ist es auszuführen?), der Selbstverstärkung (Prima, das hat gut geklappt!) und der Selbstbewertung des Ausgangs und der Handlung (Recht so! Das andere könnte ich aber noch verbessern!). Diese Elemente können zu unterschiedlichen Zeitpunkten bzw. Phasen angewandt werden.

Die Wirksamkeit von Selbstinstruktionstraining wurde im Bereich Sport u.a. von Hackfort und Schwenkmezger (1980), Mace und Caroll (1985), Schack (1997) und Hatzigeorgiadis et al. (2009) nachgewiesen. Des Weiteren stellt es ein weit verbreitetes und anerkanntes Verfahren im Sport, speziell im Klettersport, dar (Schädle-Schardt, 2002). Aufgrund seiner unangefochtenen Rolle im Bereich der Angstbewältigungsstrategien und nicht zuletzt auf Grund der positiven Ergebnisse von Mace und Carroll (1985) wird angenommen, dass das Selbstinstruktionstraining einen größeren Einfluss auf die Angst besitzt als die Atemübung.

\section{Methode}

\section{Versuchspersonen}

36 Versuchspersonen, 12 männliche und 24 weibliche, im Alter zwischen 19 und 29 Jahren, erklärten sich freiwillig bereit, an der Studie teilzunehmen. Die Versuchspersonen hatten keinerlei Vorerfahrungen im Bereich Klettern und waren gegenüber dem Gegenstand der Untersuchung unwissend. Die Zustandsangst wurde - wie in vielen Untersuchungen zur Angst beim Klettern (Nieuwenhuys et al., 2008; Pijpers et al., 2003) - mit der A-Trait Skala des StateTrait Angstinventars (Spielberger, Gorsuch \& Lushene, 1970), hier in der deutschen Version von Laux et al. (1981), gemessen. Sportbezogene Fragebögen fokussieren meist auf die Wettkampfangst und sind daher bei Sportanfängern in Übungssituationen nicht adäquat (vgl. Künzell \& Müller, 2008; Pijpers et al., 2003). Die Versuchspersonen wurden parallelisiert nach Geschlecht und STAI-Wert auf drei Gruppen verteilt: Eine Atemübung-Gruppe (ATG), eine Selbstinstruktion-Gruppe (SIG) und eine Kontrollgruppe (KG). Der Trait-Anxiety Durchschnittswert der ATG lag bei $M=37,6(S D=7,12)$, bei der SIG bei $M=37,9(S D=7,6)$ und bei der KG bei $M=36,8$ $(S D=6,88)$. Jede Versuchsgruppe bestand aus vier männlichen und acht weiblichen Versuchsteilnehmern.

\section{Durchführung}

Die Versuchspersonen kletterten an einem Kletterbunker eine vorgegebene Route des Schwierigkeitsgrades $3+$ auf der UIAA Skala. Die Höhe des Kletterbunkers bis zum Umlenker betrug $14 \mathrm{~m}$, die zu kletternde Strecke $15 \mathrm{~m}$. Die Kletterroute bestand aus 46 gut greif- und tretbaren Griffen und wurde in drei gleich lange und annähernd gleich schwere Zonen unterteilt.

Alle Versuchspersonen trugen gut sitzende Kletterschuhe der Firmen Roc'terra und Mammut und einen Klettergurt der Firma Petzl. Gesichert wurden die Versuchsteilnehmer über die Toprope Sicherungstechnik. Hierzu wurde ein Einfachseil der Firma Edelrid sowie ein ATC Sicherungsgerät der Firma Black Diamond benutzt. Gesichert wurde von einem erfahrenen Kletterer.

\section{Treatment}

\section{Atemübung-Gruppe (ATG)}

Den Vpn der ATG wurden der Pulsmesser und der Klettergurt angelegt und die Kletterschuhe angezogen. Sie wurden in das Sicherungsseil eingebunden und setzten sich dann zwei Meter vor die Kletterroute auf einen bequemen Campingsessel. Dort wurde eine kurze Atemübung durchgeführt (nach Lichstein, 1988, S. 164):

„Bevor es losgeht, schließe die Augen und höre gut zu. Atme tief ein. Halte nun deinen Atem für 5 bis 10 Sekunden an und atme dann langsam und ruhig aus (Pause bis die Exhalation beginnt). Fühle, wie sich alle Verspannung löst und aus deinem ganzen Körper herausströmt wenn du ausatmest (Pause bis die Vpn für den nächsten tiefen Atemzug bereit ist). Atme noch einmal tief ein und halte deinen Atem 5 bis 10 Sekunden an (Pause bis Exhalation beginnt). Fühle, wie sich dein Körper beim langsamen Ausatmen mehr und mehr entspannt. Atme nun noch viermal für dich selber tief ein und ganz langsam wieder aus und fühle, wie sich dein Körper mehr und mehr entspannt. Wenn du dich bereit und entspannt fühlst, darfst du aufstehen und losklettern."

Anschließend füllten die Versuchspersonen das erste Anxiety Thermometer aus und kletterten los.

\section{Selbstinstruktions-Gruppe (SIG)}

Den Versuchsteilnehmern der SIG wurden zuerst der Pulsmesser und der Klettergurt angelegt und sie wurden in das Sicherungsseil eingebunden. Um eine Grund- 
lage für die Selbstinstruktionen zu liefern, wurden ihnen die Funktion und die Sicherheitsnormen des Materials anschaulich gemacht („Das Seil, das wir hier benutzen, kann Belastungen von bis zu $9 \mathrm{KN}$ standhalten, d.h. man könnte auch einen Kleinwagen an das Seil hängen“). Für die Entwicklung der Selbstinstruktionen wurde ein von Meichenbaum (1977b, S. 424) entwickeltes Vorgehen genutzt und auf die kletterspezifische Situation abgestimmt. Hierbei wurden vier Phasen unterschieden: Vorbereitung auf den Stressor, Konfrontation und Umgang mit dem Stressor, Bewältigung des Gefühls übermannt zu werden und Verstärkung durch Selbstaussagen. Um den Versuchspersonen eine Einführung in das Selbstinstruktionstraining und eine Vorstellung der Anwendung beim Klettern zu geben, wurden ihnen Beispiele für Selbstinstruktionen gegeben („Ich schaffe es, es ist nicht mehr weit", „Der Sichernde ist groß und stark und lässt mich nicht fallen“, ,Hier kann man ein Auto hochziehen und das Seil reißt nicht"). Danach füllten die Versuchspersonen das erste Anxiety Thermometer aus und kletterten los.

\section{Kontrollgruppe (KG)}

Den Versuchspersonen der KG wurden die Angaben zur Funktion und zu den Sicherheitsnormen des Materials nicht gegeben. Sie wurden nach dem Anlegen des Gurtes, der Kletterschuhe, des Pulsmessers und dem Einbinden ohne weitere Anweisungen aufgefordert, die Route hochzuklettern. Auch hier mussten die Versuchspersonen vor dem Losklettern das erste Anxiety Thermometer ausfüllen.

\section{Abhängige Variablen}

In früheren Untersuchungen zur Angst beim Klettern hat es sich bewährt, das Angstthermometer (AT) als Indikator für die empfundene Angst zu verwenden. Darüber hinaus wurden als physiologischen Indikator die Herzfrequenz und als Verhaltensindikator die Kletterzeit verwendet (Nieuwenhuys et al., 2008; Oudejans \& Pijpers, 2009, 2010; Pijpers, Oudejans \& Bakker, 2005; Pijpers, Oudejans \& Bakker, 2007; Pijpers, Oudejans, Bakker \& Beek, 2006; Pijpers et al., 2003).

Das AT wurde von Houtman und Bakker (1989) validiert. Auf einer $10 \mathrm{~cm}$ langen Linie, deren Anfang „überhaupt nicht ängstlich“ und deren Ende „extrem ängstlich" bedeutet, soll ein Kreuz an der Stelle gemacht werden, die den aktuellen Angstzustand repräsentiert. Die Teilnehmer mussten in den drei verschiedenen Zonen sowie vor und nach dem Klettern jeweils das AT ausfüllen, um ihren momentanen $\mathrm{Zu}$ stand zu dokumentieren. Während des Kletterns wurde die Herzfrequenz im fünf Sekunden Takt mit Hilfe einer Pulsmessuhr (Polar S725 X) aufgezeichnet. Die Kletterzeit wurde in jedem Abschnitt mit einer Stoppuhr gemessen.

Die Zustandsangst wurde an fünf Messzeitpunkten (vor dem Losklettern, nach dem Durchklettern der Abschnitte 1, 2, 3 und nach dem Ablassen), die Herzfrequenz an sechs Messzeitpunkten (vor der Intervention, am Ende der Intervention, vor dem Losklettern und nach dem Durchklettern der Abschnitte 1, 2, 3), die Kletterzeit an drei Messzeitpunkten (nach dem Durchklettern der Abschnitte 1, 2, 3) erhoben.

\section{Ergebnisse}

\section{Anxiety Thermometer}

Die mit dem Anxiety Thermometer gemessen Werte steigen stetig mit zunehmender Höhe (vgl. Tab. 1).

In einer $3 \times 4$ ANOVA mit dem Zwischensubjektfaktor Gruppe (ATG, SIG, KG) und dem Messwiederholungsfaktor Zeitpunkt (vor dem Losklettern, nach Durchklettern der Stufe 1, 2 und 3, vgl. Abb. 1) wird der Effekt des Messwiederholungsfaktors hoch

Tabelle 1. Mittelwerte und Standardabweichungen der Werte des Angstthermometers der Gruppen zu den unterschiedlichen Messzeitpunkten. 0 bedeutet „überhaupt nicht ängstlich“, 10 bedeutet „,extrem ängstlich“

\begin{tabular}{|c|c|c|c|c|c|c|}
\hline & \multicolumn{6}{|c|}{ Gruppe } \\
\hline & \multicolumn{2}{|c|}{ Atemübung } & \multicolumn{2}{|c|}{ Selbstinstruktion } & \multicolumn{2}{|c|}{ Kontrolle } \\
\hline & $M$ & $S D$ & $M$ & $S D$ & $M$ & $S D$ \\
\hline AT beim Losklettern & 2,0 & 1,4 & 3,5 & 2,1 & 2,8 & 1,9 \\
\hline AT nach Zone 1 & 2,6 & 2,0 & 3,9 & 2,6 & 4,1 & 2,1 \\
\hline AT nach Zone 2 & 4,8 & 2,1 & 5,2 & 2,4 & 4,9 & 2,6 \\
\hline AT nach Zone 3 & 5,8 & 1,7 & 5,9 & 3,0 & 5,7 & 3,4 \\
\hline
\end{tabular}




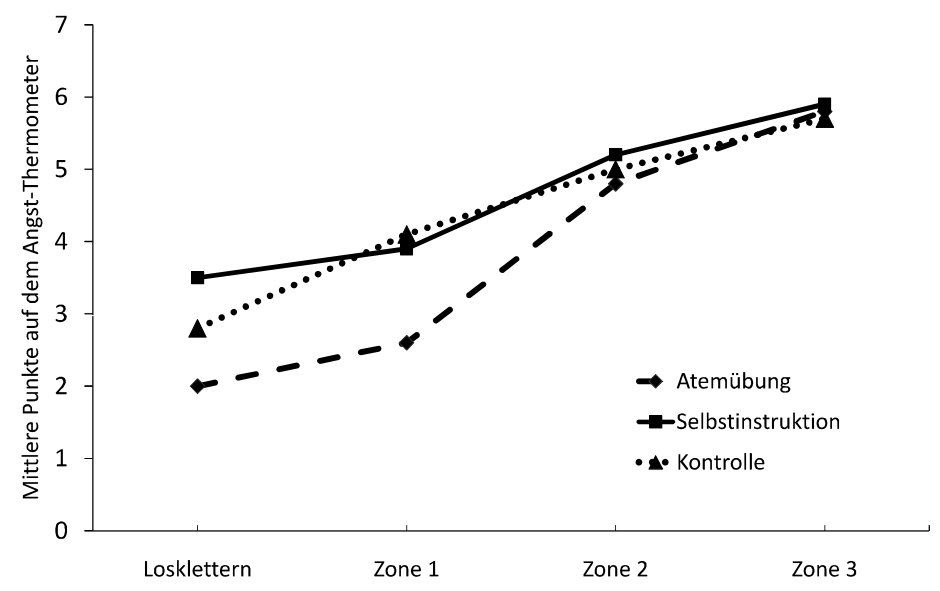

Abbildung 1. Ergebnisse der Angstmessung mit dem Angstthermometer beim Losklettern und nach dem Durchsteigen der verschiedenen Kletterzonen bei den beiden Treatmentgruppen und der Kontrollgruppe. Die Skala reicht von 0 bis 10, wobei höhere Werte eine höhere Angst bedeuten.

signifikant $(F(1.99 ; 65.73)=37.175, p<0.001$, $\eta_{\text {part }}^{2}=0.745$, Korrektur der Freiheitsgrade nach Greenhouse-Geisser).

Unterschiede zwischen den Gruppen $(F(2.33)<1$ sowie in der Interaktion zwischen den Gruppen und der Werte des AT $(F(4.0 ; 65.7)=1.19 ; p=0.323$, Korrektur der Freiheitsgrade nach Greenhouse-Geisser)) sind hingegen vermutlich zufällig bedingt.

\section{Herzfrequenz}

Die Herzfrequenz ist wesentlich beeinflusst von der physischen Belastung beim Klettern. Sie wird trotzdem erhoben und berichtet, weil sie zum einen als eine Kontrolle für die Wirksamkeit der Instruktionen dienen kann, zum zweiten es auch unter der für alle
Vpn gleichen physischen Belastung zwischen den Gruppen Unterschiede geben könnte, die dann auf die psychische Belastung durch Angst zurückgeführt werden könnte.

Ein Hinweis auf die Wirksamkeit der Intervention kann der Vergleich der Herzfrequenz vor der Instruktion, unmittelbar danach und direkt vor dem Losklettern geben (vgl. Abb. 1, Messzeitpunkte ,,vor Intervention“, „nach Intervention“", ,vor Losklettern"). Ein $3 \times 2$ ANOVA mit dem Messwiederholungsfaktor Messzeitpunkt (vor, nach, losklettern) und dem Faktor Gruppe (ATG, SIG) ergibt einen hoch signifikanten Unterschied zwischen den Messzeitpunkten $\left(F(2.44)=64.24, p<0.0005, \eta_{\text {part }}^{2}=\right.$ 0.745). Die paarweisen Vergleiche zwischen den Messzeitpunkten (post hoc, Anpassung für Mehrfachvergleiche nach Bonferroni) ergeben wechselseitig hoch signifikante Unterschiede zwischen allen drei Messzeitpunkten, wobei direkt vor dem Klettern die höchsten, direkt nach der Intervention die niedrigsten Herzfrequenzen gemessen werden. Die beiden Treatmentgruppen unterscheiden sich nicht. Vor dem Losklettern hat - nicht hypothesenkonform - die Kontrollgruppe eine geringere Herzfrequenz als die beiden Treatmentgruppen. Während des Kletterns steigt mit der Kletterhöhe die Herzfrequenz stetig an.

Eine $3 \times 4$ ANOVA mit dem Zwischensubjektfaktor Gruppe (ATG, SIG, KG) und dem Messwiederholungsfaktor Zeitpunkt (vor dem Losklettern, nach Durchklettern der Stufe 1, 2 und 3) ergibt einen sigifikanten Messwiederholungseffekt $(F(1.75 ; 57.76)=$ $\left.654.12 ; p<0.0005 ; \eta_{\text {part }}^{2}=0.952\right)$ sowie eine signifikante Interaktion zwischen Gruppe und Zeitpunkt $\left(F(3.5 ; 57.76)=3.318 ; p=0.20 ; \eta_{\text {part }}^{2}=0.167\right.$, Korrekturen der Freiheitsgrade jeweils nach GreenhouseGeisser).

Tabelle 2. Mittelwerte und Standardabweichungen der Herzfrequenzen der Gruppen zu den unterschiedlichen Messzeitpunkten

\begin{tabular}{|c|c|c|c|c|c|c|}
\hline & \multicolumn{6}{|c|}{ Gruppe } \\
\hline & \multicolumn{2}{|c|}{ Atemübung } & \multicolumn{2}{|c|}{ Selbstinstruktion } & \multicolumn{2}{|c|}{ Kontrolle } \\
\hline & $M$ & $S D$ & $M$ & $S D$ & $M$ & $S D$ \\
\hline HF vor Treatment & 96,3 & 13,5 & 88,8 & 13,7 & 92,1 & 8,9 \\
\hline HF nach Treatment & 79,1 & 12,6 & 75,6 & 8,8 & / & / \\
\hline HF losklettern & 104,6 & 16,1 & 98,5 & 11,0 & 90,3 & 13,5 \\
\hline HF nach Zone 1 & 152,1 & 15,7 & 143,5 & 18,2 & 149,8 & 12,6 \\
\hline HF nach Zone 2 & 160,9 & 11,4 & 159,8 & 14,6 & 159,9 & 16,4 \\
\hline HF nach Zone 3 & 169,0 & 11,2 & 168,5 & 14,1 & 167,7 & 12,8 \\
\hline
\end{tabular}




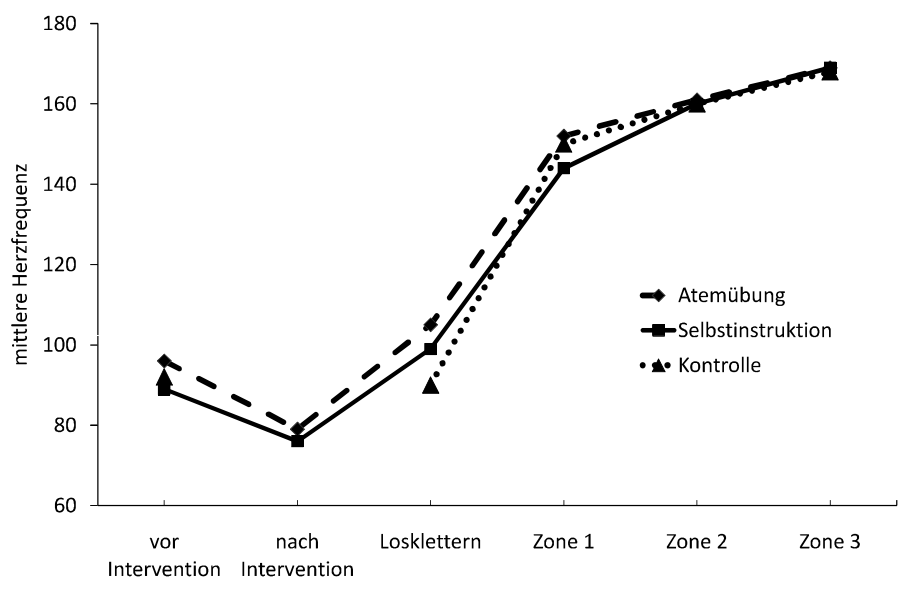

Abbildung 2. Ergebnisse der Herzfrequenzmessung vor und nach der Intervention, beim Losklettern und nach dem Durchsteigen der verschiedenen Kletterzonen bei den beiden Treatmentgruppen und der Kontrollgruppe in Schlägen pro Minute. Die Kontrollgruppe hatte keine Intervention, daher fehlt ihr Wert nach der Intervention.

\section{Kletterzeit}

Die Unterschiede in den Kletterzeiten (vgl. Tabelle 3) zwischen den verschiedenen Gruppen sind nicht signifikant $(F(2.33)=1.76, p=0.188)$ die Kletterzeit nimmt wie erwartet in den Zonen zunehmender Höhe zu $(F(2.66)=57.55 ; p<0.001)$. Die Zunahme der Kletterzeit ist auch zwischen den Gruppen nicht signifikant unterschiedlich $(F(4.66)<1)$.

\section{Diskussion}

Ziel der durchgeführten Untersuchung war, die Wirkung von zwei Kurzinterventionen auf die Angst beim Klettern zu erheben: Der Deep Breathing Tech- nik nach Lichstein (1988) und dem Selbstinstruktionstraining nach Meichenbaum (1977a, 1977b).

Analysiert man die Werte des Angstthermometers während des Kletterns, so zeigt sich, dass keine signifikanten Unterschiede zwischen den beiden Interventionsgruppen und der Kontrollgruppe nachgewiesen werden können. Die Interpretation von nicht signifikanten Ergebnissen birgt die Schwierigkeit, dass sie einerseits damit begründet werden können, dass die Intervention tatsächlich nicht wirksam ist, andererseits können aber Fehler bei dem Design oder der Durchführung der Untersuchung die Fehlervarianz in die Höhe treiben, so dass der untersuchte Effekt nicht wirksam werden kann. Trotz dieser Interpretationsschwierigkeiten plädieren Weinberg und Comar (1994) nachdrücklich dafür, auch Berichte zu veröffentlichen, in denen keine Wirkung einer Intervention nachgewiesen werden kann, um dem ,publication bias“" (S. 412) entgegenzuwirken.

Wir werden im Folgenden versuchen, allen Argumenten nachzugehen, die für, aber auch gegen die Interpretation sprechen, dass die untersuchten Kurzinterventionen keine Wirkung zeigen.

Alle drei erhobenen Indikatoren der Angst, also das Angstthermometer, die Kletterzeit und die Herzfrequenz, bestätigen die bekannten Befunde, dass die Angst beim Klettern mit zunehmender Höhe steigt (Pijpers et al., 2003; Scherer et al., 2010). Eine mangelnde angsteinflößende Wirkung der Kletterroute scheidet somit als Fehlerquelle aus. Schwieriger ist die Beurteilung der Qualität der Intervention. Da aus methodischen Gründen die Erhebung der Daten für das Angstthermometer während der Intervention ausscheidet, bleibt die Herzfrequenz als Indikator für die Qualität der Intervention. Hier zeigt sich bei beiden Treatmentgruppen ein hoch signifikantes Absinken. Daraus leiten wir ab, dass die Intervention eine beru-

Tabelle 3. Mittelwerte und Standardabweichungen der Kletterzeiten der Gruppen zu den unterschiedlichen Messzeitpunkten

\begin{tabular}{|c|c|c|c|c|c|c|}
\hline & \multicolumn{6}{|c|}{ Gruppe } \\
\hline & \multicolumn{2}{|c|}{ Atemübung } & \multicolumn{2}{|c|}{ Selbstinstruktion } & \multicolumn{2}{|c|}{ Kontrolle } \\
\hline & $M$ & $S D$ & $M$ & $S D$ & $M$ & $S D$ \\
\hline Zeit für Zone 1 & $0: 47,5$ & $0: 22,6$ & $0: 51,0$ & $0: 18,2$ & $1: 03,5$ & $0: 26,3$ \\
\hline Zeit für Zone 2 & $1: 19,8$ & $0: 19,9$ & $1: 21,8$ & $0: 31,5$ & $1: 48,9$ & $0: 56,7$ \\
\hline Zeit für Zone 3 & $1: 32,5$ & $0: 28,4$ & $1: 46,5$ & $0: 48,4$ & $1: 57,3$ & $0: 46,7$ \\
\hline Gesamtkletterzeit & $3: 39,9$ & $0: 55,2$ & $3: 59,3$ & $1: 33,2$ & $4: 49,7$ & $2: 01,9$ \\
\hline
\end{tabular}


higende und damit auch angstreduzierende Wirkung hatte.

Kritisch hinterfragt werden müssen die Auswirkungen des Fehlens einer Placebo-Behandlung der Kontrollgruppe (Vealey, 1994). „Control groups should also be motivated, allowing individuals to feel that they are also being given special attention and are doing something that they feel is worthwhile" (Weinberg \& Comar, 1994, S. 413). In dieser Studie haben wir auf eine Placebo-Intervention verzichtet. Dies erscheint uns aus zwei Gründen legitim: Erstens wussten die Vpn der Kontrollgruppe nicht, dass sie in der Kontrollgruppe waren, sondern sie gingen davon aus, dass sie in der Versuchsgruppe zur Bestimmung der Angst beim Klettern waren. Sie konnten die angewendeten Interventionen bei Vpn der anderen Gruppen nicht beobachten, da alle Versuchspersonen einzeln $\mathrm{zu}$ verschiedenen Terminen kletterten. Zweitens ergibt es sich aus der Logik des Kletterns, dass auch Versuchspersonen der Kontrollgruppe ,spezielle Aufmerksamkeit" gewidmet wird. Ihnen wird beim Anlegen des Klettermaterials geholfen, sie werden motiviert, die gesamte Route zu durchsteigen und nicht vorzeitig abzubrechen und schließlich werden sie die gesamte Zeit über aufmerksam gesichert. Insofern schließen wir aus, dass sich die Versuchspersonen der Kontrollgruppe als „Versuchspersonen 2. Klasse“ empfanden. Aber, selbst wenn dem so sein sollte, würde eine fehlende Placebo-Intervention die beobachteten Effekte der Interventionen verstärken. Die beobachtete Wirkungslosigkeit der Kurzinterventionen hingegen verliert nichts an ihrer Aussagekraft.

Die beruhigende Wirkung der Intervention schlägt in das Gegenteil um, wenn mit dem Klettern begonnen werden soll. Hier ist die Herzfrequenz noch höher als die Ruhefrequenz vor dem Beginn der Intervention, sie liegt bei den beiden Treatmentgruppen höher als bei der Kontrollgruppe. Post-hoc könnte man vermuten, dass durch die Intervention die Versuchspersonen erst darauf hingewiesen wurden, dass die folgende Kletterpartie bedrohlich sein könnte, woraufhin ihre Herzfrequenz steigt. Diese Interpretation wird durch die subjektiven Daten des Angstthermometers allerdings nicht bestätigt, aber auch nicht widerlegt, da die dort gefundenen Unterschiede vermutlich zufälliger Natur sind.

Der Hauptunterschied zu den oben genannten Studien, in denen die Wirksamkeit der Entspannungsverfahren durch Atemübungen oder durch Selbstinstruktion nachgewiesen wurde, liegt in der fehlenden Übung des jeweiligen Verfahrens. In dieser Studie wurden diese Verfahren ohne weitere Vorbereitung und ohne jedes Training angewendet. Dies scheint jedoch für die Wirksamkeit nötig zu sein. So fordert Meichenbaum (1977a) für den klinischen Bereich der
Angsttherapie, dass die Selbstinstruktionstechnik von Klient und Therapeut gemeinsam erarbeitet werden muss. Ein vorgefertigtes Verfahren, wie es in dieser Studie angewendet wurde, hat offenbar keine Wirkung. Für den Bereich der Sportpsychologie bedeutet dies, dass angstreduzierende Interventionen offenbar nicht ,von der Stange“ zu haben sind, sondern dass ihre Wirksamkeit nur durch eine sorgfältig vorbereitetes Training gewährleistet werden kann, das die Athletin und der Trainer oder die Sportpsychologin gemeinsam erarbeiten.

Zusammenfassend kann festgestellt werden, dass bei nur kurzfristig angeleiteten somatischen oder kognitiven Interventionen zur Angstreduktion keine Wirkung nachgewiesen werden konnte. Wirksame Techniken zur Angstreduktion bedürfen offenbar einer sorgfältigen Vorbereitung. Wenn Angstreduktionstechniken ohne großen Zeitaufwand kurzfristig durchgeführt werden, scheint es so zu sein, dass sie gerade auf die Bedrohung aufmerksam machen. Bevor man also dies tut, sollte man die Kletteranfänger lieber gleich ,ins kalte Wasser schmeißen“.

\section{Literatur}

Baumann, S. (2006). Psychologie im Sport (4., überarb. Aufl.). Aachen: Meyer \& Meyer.

Bell, J. \& Saltikov, J. (2000). Mitchell's relaxation technique: Is it effective? Physiotherapy, 86, 473-478.

Garssen, B., De Ruiter, C. \& Van Dyke, R. (1992). Breathing retraining. A rational placebo? Clinical Psychology Review, 12, 141-153.

Hackfort, D. \& Schwenkmezger, P. (1980). Angst und Angstkontrolle im Sport. Köln: bps.

Hatzigeorgiadis, A., Zourbanos, N., Mpoumpaki, S. \& Theodorakis, Y. (2009). Mechanisms underlying the self-talk-performance relationship: The effects of motivational self-talk on self-confidence and anxiety. Psychology of Sport and Exercise, 10, 186-192.

Houtman, I. \& Bakker, F. C. (1989). The anxiety thermometer: A validation study. Journal of Personality Assessment, 53, 575-582.

Künzell, S. \& Müller, J. (2008). The use of bigfoots reduces state anxiety in novice skiers. Journal of Applied Sport Psychology, 20, 253-260.

Laux, L., Glanzmann, P., Schaffner, P. \& Spielberger, C. D. (1981). Das State-Trait-Angstinventar. Weinheim: Beltz Testgesellschaft.

Lazarus-Mainka, G. \& Siebeneick, S. (2000). Angst und Ängstlichkeit. Göttingen: Hogrefe.

Lazarus, R. S. (1984). On the primacy of cognition. American Psychologist, 39, 124-129.

LeDoux, J. (1997). The emotional brain. New York: Simon \& Schuster.

Lichstein, K. L. (1988). Clinical Relaxation strategies. New York: Wiley.

Lum, L. (1983). Physiological considerations in the treatment of hyperventilation syndromes. Journal of Drug Research, 8, 1867-1872. 
Mace, R. D. \& Carroll, D. (1985). The control of anxiety in sport: stress inoculation training prior to abseiling. International Journal of Sport Psychology, 16, 165175.

Meichenbaum, D. (1977a). Cognitive behavior modification: an integrative approach. New York, NY: Plenum Press.

Meichenbaum, D. (1977b). Methoden der Selbstinstruktion. In F. H. Kanfer \& L. G. Buckner (Hrsg.), Möglichkeiten der Verhaltensänderung (S. 407-451). München: Urban \& Schwarzenberg.

Munzert, J. (1997). Sprache und Bewegungsorganisation. Schorndorf: Hofmann.

Myers, D. G. (2008). Psychologie (2. Aufl.). Heidelberg: Springer.

Nieuwenhuys, A., Pijpers, J. R., Oudejans, R. R. D. \& Bakker, F. C. (2008). The influence of anxiety on visual attention in climbing. Journal of Sport \& Exercise Psychology, 30, 171-185.

Olschewski, A. (1995). Atementspannung: Abbau emotionaler und körperlicher Anspannung durch Atemtherapie. Heidelberg: Haug.

Oudejans, R. R. D. \& Pijpers, J. R. (2009). Training with anxiety has a positive effect on expert perceptual-motor performance under pressure. Quarterly Journal of Experimental Psychology, 62, 1631-1647.

Oudejans, R. R.. D. \& Pijpers, J. R. (2010). Training with mild anxiety may prevent choking under higher levels of anxiety. Psychology of Sport and Exercise, 11, 44-50.

Payne, R. (2004). Relaxation techniques. In G. Kolt \& M. Andersen (Eds.), Psychology in the Physical and Manual Therapies (pp. 111-125). Edinburgh: Churchill Livingstone.

Pijpers, J. R., Oudejans, R. R. D. \& Bakker, F. C. (2005). Anxiety-induced changes in movement behaviour during the execution of a complex whole-body task. The Quarterly Journal of Experimental Psychology: Section A, 58, 421-445.

Pijpers, J. R., Oudejans, R. R. D. \& Bakker, F. C. (2007). Changes in the perception of action possibilities while climbing to fatigue on a climbing wall. Journal of Sports Sciences, 25, 97-110.

Pijpers, J. R., Oudejans, R. R. D., Bakker, F. C. \& Beek, P. J. (2006). The role of anxiety in perceiving and realizing affordances. Ecological Psychology, 18, 131-161.
Pijpers, J. R., Oudejans, R. R. D., Holsheimer, F. \& Bakker, F. C. (2003). Anxiety-performance relationships in climbing: A process-oriented approach. Psychology of Sport and Exercise, 4, 283-304.

Schack, T. (1997). Ängstliche Schüler im Sport: Interventionsverfahren zur Entwicklung der Handlungskontrolle. Schorndorf: Hofmann.

Schädle-Schardt, W. (2002). Klettern: Lehren - Lernen Erleben. Aachen: Meyer und Meyer.

Scherer, H.-G., Kuhn, M., Henn, A., \& Zepig, R. (2010). Der „weiche Griff“ beim Klettern. In G. Amesberger, T. Finkenzeller \& S. Würth (Hrsg.), Psychophysiologie im Sport - zwischen Experiment und Handlungsoptimierung (S. 157). Hamburg: Feldhaus.

Spielberger, C. D., Gorsuch, R. L. \& Lushene, R. E. (1970). Manual for the State-Trait Anxiety Inventory. Palo Alto, California: Consulting Psychologist Press.

Sudsuang, R., Chentanez, V. \& Veluvan, K. (1991). Effect of Buddhist meditation on serum cortisol and total protein levels, blood pressure, pulse rate, lung volume and reaction time. Physiology and Behavior, 50, 543-548.

van Reekum, C. M., Urry, H. L., Johnstone, T., Thurow, M. E., Frye, C. J., Jackson, C. A. et al. (2007). Individual differences in amygdala and ventromedial prefrontal cortex activity are associated with evaluation speed and psychological well-being. Journal of Cognitive Neuroscience, 19, 237-248.

Vealey, R. S. (1994). Current status and prominent issues in sport psychology interventions. Medicine and Science in Sports and Exercise, 26, 495-502.

Weinberg, R. S., \& Comar, W. (1994). The effectiveness of psychological interventions in competitive sport. Sports Medicine, 16, 406-418.

Zajonc, R. B. (1984). On the primacy of affect. American Psychologist, 39, 117-123.

Stefan Künzell und Vanessa Mann

Institut für Sportwissenschaft

Justus-Liebig-Universität Gießen

Kugelberg 62

35394 Gießen

E-Mail: stefan.kuenzell@sport.uni-giessen.de 